\title{
Mono-static Automotive Joint Radar-Communications System
}

\author{
Sayed Hossein Dokhanchi*, Mohammad Alaee-Kerahroodi*, Bhavani Shankar Mysore R*, \\ and Björn Ottersten* \\ ${ }^{*}$ SnT, University of Luxembourg.
}

\begin{abstract}
The paper investigates the applicability of a conventional communications signal for sensing and detection in vehicular scenario. This Joint RadarCommunications (JRC) system caters to an automotive setting characterized by a multi-target environment including clutter. In this contribution, an automotive radar employing phase modulated communication signal is considered wherein the detection of other vehicles is enabled in presence of clutter. Particularly, it is shown when the clutter is dominant in the environment, bit error rate (BER) of communications will suffer. Further, the receiver processing includes demodulation of the communication symbols at communicating vehicle in addition to the extraction of the radar parameters - range and Doppler shifts of the targets - at the JRC-equipped vehicle. The study investigates the impact of various parameters in determining the performance of the JRC set-up.
\end{abstract}

Index Terms-Intelligent transportation system, Joint radar-communications system, Radar signal processing.

\section{INTRODUCTION}

Automotive radar systems employ various portions of the radio spectrum to detect obstacles such as vehicles or pedestrians, e.g, 77-81 GHz. Radars can alert the driver of possible collisions, automatically trigger safety measures and ultimately undertake manoeuvres to avoid or mitigate collisions. On the other hand, future vehicles will be connected as communication is seen as an enabler for enhanced level of safety to the road transport system. Consequently, intelligent transportation systems (ITS) aim at low-cost, compact and efficient sensing and communication devices by combining common resources of different sensors in a single platform [1], [2]. Such a system, referred to as vehicular joint radar-communications (JRC), enables the sensing and communication systems to share spectrum for operations and hardware resources.

Realizing the importance of JRC, recent works [3], [4], [5], [6], [7], [8], [9], have investigated various ways of multiplexing radar and communications in different dimensions, i.e., time, frequency, space and code. These work assume no prior knowledge about the target scene.

The authors have studied a bi-static JRC waveform in [5] modulating the existing structure of phasemodulated continuous waveform (PMCW) to support additional functionality of data transmission. An orthogonal frequency-division multiple access (OFDMA) based automotive bi-static JRC waveform has been developed in [6]; here the system associates part of OFDMA subcarriers for radar processing and the rest for enabling JRC functionalities. However, these works encounter the issue of lack of degrees of freedom in a bi-static JRC waveform design to estimate communication symbols in addition to the ranges, Doppler shifts and angle of arrivals of the targets of interest. In [9], the authors corroborate this observation through identifiability analysis and have developed the Cramér-Rao lower bound (CRLB) on JRC parameters (range, angle, Doppler and communication). Identifiability issues are solved either by reserving a portion of resources exclusively for one functionality or by considering the multicarrier phase modulated continuous waveform (MC-PMCW) [7].

Towards quantifying the advantages of JRC systems and aid in their design, several works have studied the performance analysis of such systems. In [10], the authors have derived bounds on performance of the JRC in terms of data information rate for communications and the receiver operating characteristic (ROC) curve for radar. The authors have introduced the estimation rate which is a metric analogous to the communications data rate [11]. It further enunciates the difference in priorities among the bits corresponding to the two rates. Based on a novel radar estimation information rate, [12] has investigated achievable bounds on performance for a receiver that observes communications and radar return in the same frequency allocation, operating in a single joint system. In addition to the aforementioned investigations, several works have considered optimization of the waveform in different scenarios towards enhancing the identified radar tasks and communication performance [3], [4], [13], [14]. Central to these works is the apriori knowledge on clutter/channel state information (CSI).

A key motivation behind our proposed joint system design is to enable the detection of other vehicles through a phase-modulated communication signal wherein no CSI is required. This is in contrast to [3], [4], [13], [14] for which apriori knowledge on CSI is essential at the transmitter. Further, in this scenario clutter is also considered unlike [5]- [7] and is shown that the bit error rate (BER) of communications increases when the clutter is strong. Furthermore, the receiver processing includes demodulation of the communication symbols 
at the appropriate receiver in addition to the extraction of the range and Doppler shifts of the targets at the JRCequipped vehicle. The performed study demonstrates the impact of the joint communications and radar set-up on the performance of two systems in terms of BER and root mean square error (RMSE), respectively.

This paper investigates the design of a mono-static JRC system to convey both radar and communications information while avoiding mutual interference in a dynamic automotive environment. The contributions include,

- A JRC system is developed based on a communications waveform with PSK modulation where both systems operate simultaneously in time, frequency and space including the effect of clutter,

- Receiver processing (matched-filter following by a FFT-beam-scanner) is devised for detection of the radar parameters and a PSK-demodulator for communications to ensure robustness of joint design to interference,

- The performance of proposed waveform in terms of RMSE for radar and BER of communications is analyzed.

Organization: Section II introduces the scenario, signal and system model and transmitter design. Section III includes the proposed receiver processing techniques for the JRC system. Simulation results are provided in section IV and conclusions drawn in section V.

\section{Signal AND System Model}

In this section, we first introduce the considered scenario. We then propose transmit waveform design following by the model of the probing signal.

a) Automotive scenario: We consider an automotive scenario depicted in Fig. 1 where the JRC-equipped vehicle aims to communicate to the receiver of the red car. Simultaneously, from the reflections of this communications waveform, the JRC vehicle estimates the range and velocity of the other cars in the target scene in the presence of clutter. To focus on the JRC waveform design, many imperfections including synchronization, phase noise, requirements on safety and privacy are assumed not to influence the analysis.

The system model for the considered scenario involves single transmitter and receiver in each car. A planar wave is assumed for the model [15] and the parameters of interest are ranges and Doppler shifts of targets and communication symbols. The targets are far enough to consider them as point scatterers and $Q$ number of them exist in the target scene.

\section{A. Transmitter Design}

The block diagram of the transmitter is shown in Fig. 2. The communication bits are modulated into PSK waveform with root-raised-cosine pulse shaping filter.

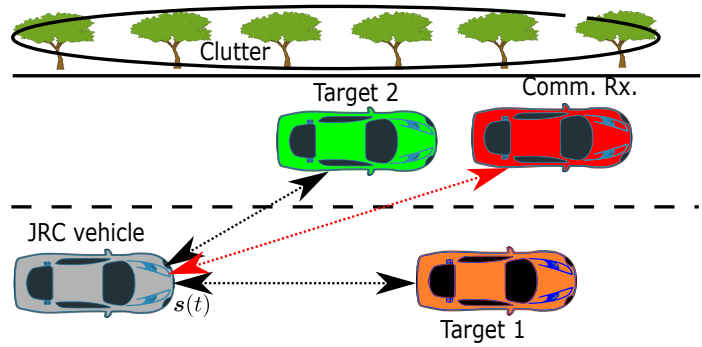

Fig. 1. Smart JRC-equipped vehicle transmits a single waveform to communicate with the communication receiver of the red car. Simultaneously, it aims to detect the range and Doppler shifts of the two other cars in the scenario that includes clutter, i.e., trees and ground.

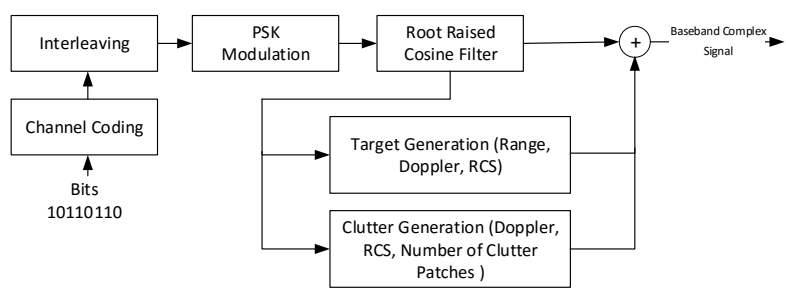

Fig. 2. Block diagram of the transmitter; Communication bits are interleaved and modulated by PSK signal, filtered by root-raised-cosine pulse shaping and transmitted over the antenna after up-conversion. Clutter is also considered in addition to the targets of interest due to presence of unwanted objects in the target scene.

The transmitter transmits $M$ repetitions of the sequence of pulses during coherent processing interval (CPI). The Doppler and flight-time is assumed to be fixed over CPI. The backscatters from $Q$ targets in addition to the clutter response impinge on receive antenna of the JRCequipped car.

The transmit signal involving PSK modulation using a pulse shaping filter $s_{i}(t)$ can be expressed as

$$
x(t)=\sum_{l=0}^{L-1} s_{i}\left(t-l t_{c}\right), 0 \leq t \leq t_{b},
$$

where $s_{i}(t)=e^{j \zeta_{i}} r c\left(\frac{t}{t_{c}}\right), \zeta_{i}$ carries the communications data. The time $t_{c}$ is symbol time, $t_{b}=L t_{c}$ is the time of transmission of a sequence of data with length $L$, and $r c$ is root-raised-cosine filter frequently used for pulse-shaping in digital modulation. This signal is upconverted to RF and transmitted over the antenna of JRC vehicle.

\section{Proposed Receive Processing}

In this section the receiver processing of the backscatters are investigated. To this end, we first develop the received signal model.

\section{A. Received Signal}

Let $R_{q}^{(1)}$ be the distance between transmitter and $q$ th target and $R_{q}^{(2)}$ be the distance between $q$ th target and 
receiver, $c$ be the speed of light, $f_{D_{q}}^{(1)}=\frac{\Delta V_{q}^{(1)}}{c} f_{c}$ and $f_{D_{q}}^{(2)}=\frac{\Delta V_{q}^{(2)}}{c} f_{c}$ be the transmit and return Doppler shifts, $\tau_{q}^{(1)}=\frac{R_{q}^{(1)}}{c}\left(\tau_{q}^{(2)}=\frac{R_{q}^{(2)}}{c}\right)$ be the forward (return) path flight-time, $h_{q}^{(1)}\left(h_{q}^{(2)}\right)$ be the accumulated effect of $q$ th target RCS and channel attenuation of $q$ th forward (return) path, $e^{j 2 \pi f_{c} \tau_{q}^{(1)}}$ be the phase shift due to signal flight-time from transmitter to target and $e^{j 2 \pi f_{c} \tau_{q}^{(2)}}$ plays the same role for target to receiver. Backscatter model at the JRC vehicle takes the form,

$$
\begin{aligned}
& y(t)=\sum_{q=0}^{Q} \sum_{m=0}^{M-1 L-1} \sum_{l=0} h_{q}^{(1)} h_{q}^{(2)} s_{i}\left(t-l t_{c}-m t_{b}-\tau_{q}^{(1)}-\tau_{q}^{(2)}\right) \\
& \times e^{j 2 \pi\left(f_{c}-f_{D_{q}}^{(1)}-f_{D_{q}}^{(2)}\right) t} e^{-j 2 \pi\left(f_{c}\left(\tau_{q}^{(2)}+\tau_{q}^{(1)}\right)+f_{D_{q}}^{(2)} \tau_{q}^{(2)}\right)} \\
& +z(t)+n(t),
\end{aligned}
$$

where $n(t)$ is the circular complex Gaussian noise, and $z(t)$ is the signal-dependent interference model defined as [16],

$$
z(t)=\sum_{q=1}^{Q_{c}} \sum_{m=0}^{M-1 L-1} \sum_{l=0}^{L-1} \alpha_{q} e^{j 2 \pi f_{D_{q}}^{(c)} t} s_{i}\left(t-l t_{c}-m t_{b}-r_{q}\right),
$$

where $\alpha_{q}, f_{D_{q}}^{(c)}$ denote the complex amplitude and normalized Doppler frequency of the $q$ th clutter, $r_{q}$ denote the delay due to $q$ th path, and $Q_{c}$ is the number of clutter scatterers.

To simplify (2), we define round-trip delay $\tau_{q}=$ $\tau_{q}^{(1)}+\tau_{q}^{(2)}$, and Doppler shifts $f_{D_{q}}=f_{D_{q}}^{(1)}+f_{D_{q}}^{(2)}$ for $q$ th scatterer and we let $e^{j \psi_{q}}=e^{-j 2 \pi\left(f_{c}\left(\tau_{q}^{(2)}+\tau_{q}^{(1)}\right)+f_{D_{q}}^{(2)} \tau_{q}^{(2)}\right)}$. After down-conversion to baseband, we can simplify (2) as

$$
\begin{aligned}
& y(t)=\sum_{q=1}^{Q} \sum_{m=0}^{M-1} \sum_{l=0}^{L-1} h_{q} e^{j \psi_{q}} e^{-j 2 \pi f_{D_{q}} t} \\
& \times s\left(t-l t_{c}-m t_{b}-\tau_{q}\right)+z(t)+n(t),
\end{aligned}
$$

where $h_{q}=h_{q}^{(1)} h_{q}^{(2)}$. We will now focus on the receiver processing structure for the JRC system.

\section{B. Receive Processing}

In this section, first a radar receiver is proposed followed by the detection of the communication symbol.

1) Radar Receiver: Fig. 3 illustrates the block diagram of the receiver indicating the processing required to obtain relevant parameters. The radar parameters are estimated from the matrix $\boldsymbol{Y} \in \mathbb{C}^{M \times L}$ which is obtained from (3) by stacking the received sequences of data with length $L$ for each of the $M$ JRC repetition frames in one Coherent Processing Interval (CPI). For range estimation, one should apply a matched-filter over each of the $M$ pulse sequences. Let $r_{m}(k)$ be the result of the

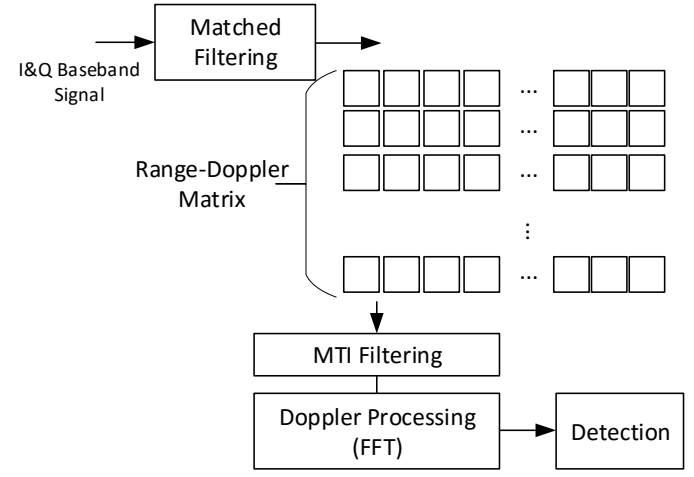

Fig. 3. Block diagram of the radar receiver; the baseband signal is matched-filtered to estimate the range of the targets followed by moving target indication (MTI) filter to mitigate the effect of clutter. Eventually, FFT-beam-scanner is applied to detect the Doppler shifts of the targets.

matched-filter for $m$ th pulse; we then obtain the delay (range) of the targets as,

$$
\hat{\tau}_{q}=\arg \max _{k}\left|r_{m}(k)\right|, 1 \leq m \leq M,
$$

where $k$ is the time index. Since range and delay are interconnected by $R_{q}=\tau_{q} c$, it can be easily obtained from (4). According to block diagram of Fig. 3 the result of the matched-filter, is used for Doppler processing. Before that, a moving target indication (MTI) is applied to eliminate the effect of clutter. We store the result of the matched-filter in matrix $\boldsymbol{V} \in \mathbb{C}^{M \times L}$, where by FFT operation is used to estimate the Doppler shifts of the targets. Towards this, we let $f=\left[\begin{array}{llll}\omega^{0} & \omega^{1} & \cdots & \omega^{M-1}\end{array}\right]^{T}$, wherein $\omega=e^{-j \frac{2 \pi}{M}}$ and denote the FFT matrix as, $\boldsymbol{F}=$ $\left[\begin{array}{lll}\boldsymbol{f}^{0^{T}} & \cdots & \boldsymbol{f}^{(M-1)^{T}}\end{array}\right]^{T} \in \mathbb{C}^{M \times M}$. Then we apply FFT operation as,

$$
\boldsymbol{b}=\boldsymbol{F} \boldsymbol{V} \in \mathbb{C}^{M \times L},
$$

where $\boldsymbol{b}$ is a vector containing $M-Q$ zeros and $Q$ non-zero elements and the positions of nonzero elements correspond to $Q$ Doppler frequency indices.

2) Symbol detection: The communication receiver at the JRC-equipped vehicle performs a communications processing to extract the transmitted data. It requires a demodulation of the PSK-modulated symbols. It is worthy to note that each row of the matrix $\boldsymbol{Y}$ contains the same data due to the repetition of the same signal $M$ times during one CPI. This helps the communication receiver to exploit available diversity gain to enhance its performance. However, in presence of severe clutter environment the BER may suffer when the signal-tonoise ratio of the communication link is poor. 

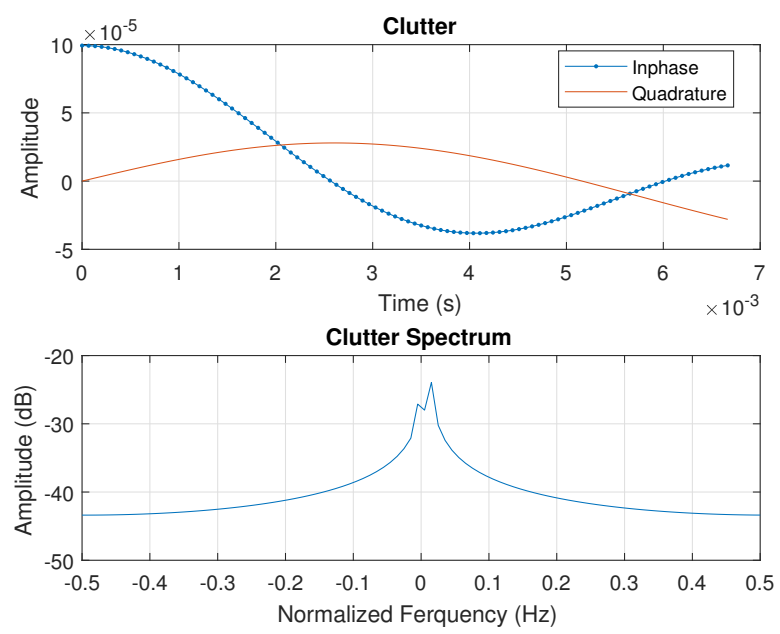

Fig. 4. Temporal and spectral characteristics of the clutter. The most spectral energy is concentrated around null frequency to present the effect of static objects on the road like trees, ground.

\section{iV. Simulation Results}

In this section, we present a numerical example to verify the functionality of our proposed method. Table I summarizes all the system parameters. The vehicular scenario considered in this section is similar to Fig. 1. An JRC-equipped vehicle is going to communicate with the communication receiver of the other car while simultaneously employing a transmit waveform to detect the two other cars in the target scene as well. The JRC vehicle transmits 25000 pulses per second, each containing 800 16-PSK modulated data. It further repeats this procedure 500 times during one CPI. The range and normalized Doppler frequency of the cars are shown in Table I. this scenario includes the effect of clutter whose temporal and spectral characteristics are illustrated in Fig. 4. The In-phase and Quadrature components of the clutter are depicted in this figure as well. Due to the static behavior of the many of the objects in a road such as ground, trees, etc., most of the spectral energy of the clutter is concentrated around zero Doppler frequency and it leaks by moving towards higher frequencies.

TABLE I

SimULATION PARAMETERS

\begin{tabular}{l|l|l}
\hline Remark & Symbol & Quantity \\
\hline Carrier frequency & $f_{c}$ & $6 \mathrm{GHz}$ \\
Bandwidth & $B W$ & $20 \mathrm{MHz}$ \\
Pulse repetition frequency & $P R F$ & $25 \mathrm{KHz}$ \\
No of symbols per pulse & $L=\frac{B W}{P R F}$ & 800 \\
No of pulses per CPI & $M$ & 500 \\
No of FFT & $N_{f}$ & $2^{12}$ \\
Modulation index & $M_{I}$ & 16 \\
Normalized Doppler shifts & $f_{D 1}, f_{D 2}$ & $0.247,-0.351$ \\
Range $R_{1}, R_{2}$ & $90 m, 164 m$ & \\
roll-off-factor of raised-cosine & $R o F$ & 0.25 \\
\hline
\end{tabular}
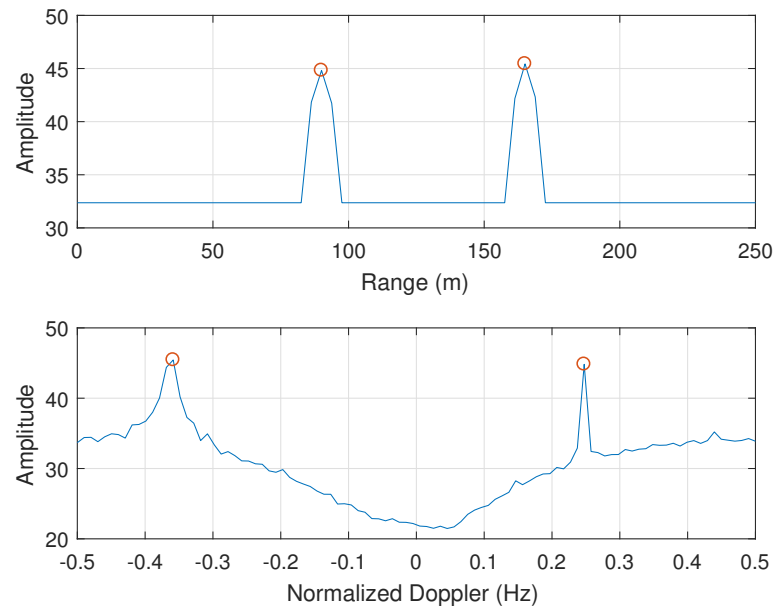

Fig. 5. The range and Doppler shifts of the two target cars: The pair of position and normalized Doppler shifts of the first and second targets are (90 $m, 0.247)$ and $(164 m,-0.351)$ respectively.

Fig. 5 shows the range and Doppler of the detected targets. The red circles in the top figure highlight the ranges of two targets at almost $90 \mathrm{~m}$ and $164 \mathrm{~m}$ and in the bottom figure they show the detected normalized Doppler frequencies of the targets at approximately $(0.247,-0.351)$. This indicates a good match between the detected and actual positions and Doppler shifts of the targets.

Fig. 6 shows the range-Doppler plane after detection of two targets at ranges-Doppler couples of (90 $m, 0.247),(164 m,-0.351)$. The locations of two targets are shown by the stars, and the small square shows the detected range-Doppler bin of the target. One observes a good match between the actual position and velocity of the targets and its estimated values.

\section{A. Radar performance}

Fig. 7 shows the root-mean-square-error (RMSE) of the estimated Doppler shifts in the presence of clutter. The figure shows that the error in Doppler estimate increases by having higher clutter to noise ratio. It also shows when the communication receiver operates at high SNR regime i.e., SNR $=30 d B$, the radar performance is higher as well. Therefore, the performance of radar and communications are bound together and mostly dependent on the SNR and clutter to noise ratio.

\section{B. Communications Performance}

Fig. 8 shows the BER of communications for various clutter to noise ratio (CNR) and several SNR values for communications. It indicates that increasing the clutter to noise ratio degrades the BER of communication significantly. It further illustrates that for higher SNR values in communications, e.g., SNR $=30 d B$ at $-30 d B$ $\mathrm{CNR}$, the BER is 0.0005 . It demonstrates that we can 


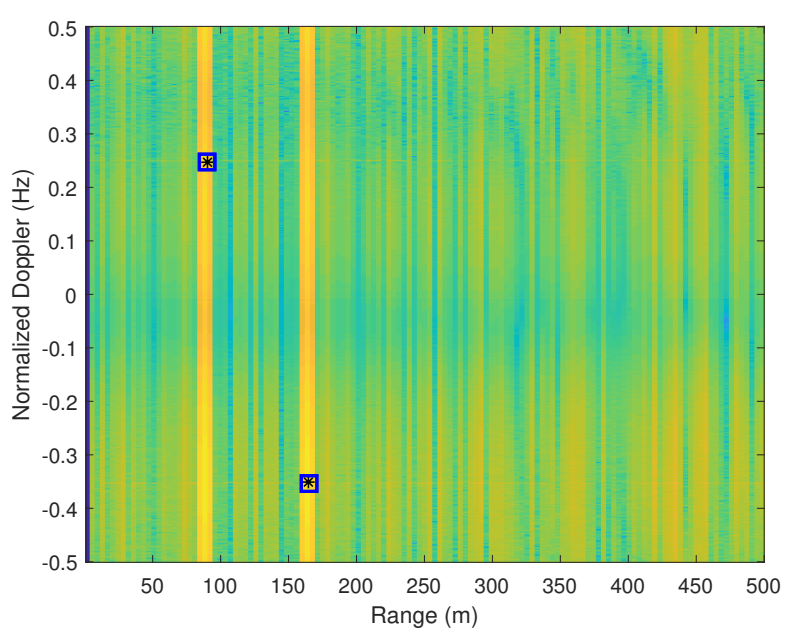

Fig. 6. Range-Doppler plane: Two targets has been detected in the range-Doppler plane, the star shows the real positions of the cars, and the square shows the estimate position. There is a good match between the estimate and real positions of the cars. The range of the targets are 90 and 164 meters, and the normalized Doppler shifts are 0.247 and -0.351 .

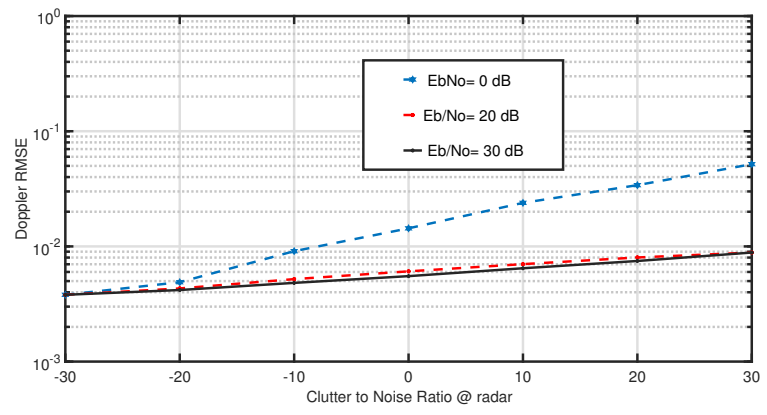

Fig. 7. RMSE of range and Doppler shifts estimate of two targets as a function of clutter to noise power ratio at various SNR conditions for communication receiver. It illustrates by increasing the clutter to noise power ratio, the error of Doppler estimates increases. It is obvious from the graph that at high SNR for communication, there is less error in estimation.

transfer data with a low probability of error within our proposed scheme. Further performance improvements can be achieved by using forward error correction codes in the communications part as well.

\section{Conclusion}

In this paper, a mono-static JRC model was considered to convey communications data simultaneously with the estimation of range and Doppler shifts of the interested targets. The scenario includes an automotive setting characterized by a multi-target environment including the clutter. Particularly, it was shown that the performance of communications system in terms of BER degrades when the clutter is strong in the environment. Further, a receiver processing was developed which includes demodulation of the communication symbols in addition

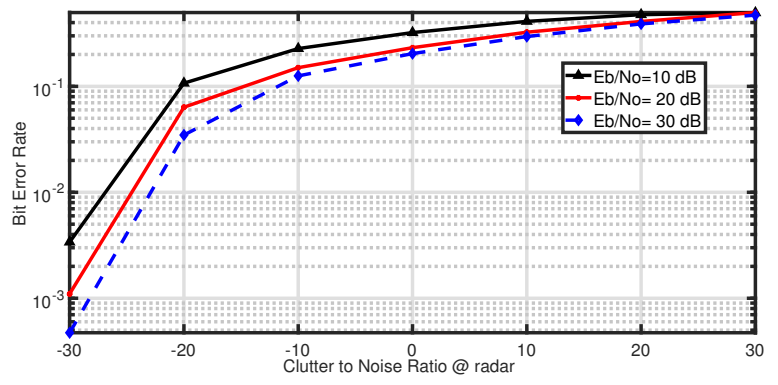

Fig. 8. The effect of clutter to noise ratio (CNR) at radar receiver on BER of the communications system for various SNR at communications receiver. It demonstrates increasing the power of clutter relative to noise has a negative impact on BER of the communications system as well. On the other hand, when the communications system is at a good SNR condition BER decreases.

to the extraction of the radar parameters at the JRCequipped vehicle. The performed study demonstrated the feasibility of the JRC system indicating the impact of the joint communications and radar set-up on the performance of two systems in terms of BER and root mean square error (RMSE), respectively.

\section{ACKNOWLEDGMENT}

This work is supported by the National Research Fund of Luxembourg under AFR-PPP grant for Ph.D. projects (Reference 11638687). The work of Dr. Mohammad Alaee-Kerahroodi was supported by FNR (Luxembourg) through the BRIDGES project Adaptive mm-Wave Radar platform for Enhanced Situational Awareness: Design and Implementation (AWARDS), CPPP17/IS/11827256/AWARDS.

\section{REFERENCES}

[1] C. Sturm and W. Wiesbeck, "Waveform design and signal processing aspects for fusion of wireless communications and radar sensing," Proceedings of the IEEE, vol. 99, no. 7, pp. 1236-1259, 2011.

[2] V. Winkler, J. Detlefsen, U. Siart, J. Buchler, and M. Wagner, "Automotive radar sensor with communication capability," in European Conference on Wireless Technologies, 2004, pp. 305308.

[3] B. Li, A. P. Petropulu, and W. Trappe, "Optimum co-design for spectrum sharing between matrix completion based MIMO radars and a MIMO communication system," IEEE Transactions on Signal Processing, vol. 64, no. 17, pp. 4562-4575, 2016.

[4] P. Kumari, J. Choi, N. Gonzlez-Prelcic, and R. W. Heath, "IEEE 802.11ad-based radar: An approach to joint vehicular communication-radar system," IEEE Transactions on Vehicular Technology, vol. 67, no. 4, pp. 3012-3027, April 2018.

[5] S. H. Dokhanchi, M. R. Bhavani Shankar, Y. A. Nijsure, T. Stifter, S. Sedighi, and B. Ottersten, "Joint automotive radarcommunications waveform design," in IEEE International Symposium on Personal, Indoor and Mobile Radio Communications, 2017.

[6] S. H. Dokhanchi, M. R. B. Shankar, T. Stifter, and B. Ottersten, "OFDM-based automotive joint radar-communication system," in 2018 IEEE Radar Conference (RadarConf18), April 2018, pp. 0902-0907. 
[7] _ - "Multicarrier phase modulated continuous waveform for automotive joint radar-communication system," in 2018 IEEE 19th International Workshop on Signal Processing Advances in Wireless Communications (SPAWC), June 2018, pp. 1-5.

[8] S. H. Dokhanchi, M. R. Bhavani Shankar, K. V. Mishra., T. Stifter, and B. Ottersten, "Performance analysis of mmwave bi-static PMCW-based automotive joint radar-communications system," in Radar Conference (RadarConf), 2019 IEEE.

[9] S. H. Dokhanchi, M. R. Bhavani Shankar, K. V. Mishra., and B. Ottersten, "A mmWave automotive joint radarcommunications system," IEEE Transactions on Aerospace and Electronic Systems, 2019.

[10] A. R. Chiriyath, B. Paul, and D. W. Bliss, "Simultaneous radar detection and communications performance with clutter mitigation," in Radar Conference (RadarConf), 2017 IEEE, 2017, pp. 0279-0284.

[11] - "Radar-communications convergence: Coexistence, cooperation, and co-design," IEEE Transactions on Cognitive Communications and Networking, vol. 3, no. 1, pp. 1-12, 2017.

[12] A. R. Chiriyath, B. Paul, G. M. Jacyna, and D. W. Bliss, "Inner bounds on performance of radar and communications coexistence." IEEE Trans. Signal Processing, vol. 64, no. 2, pp. 464-474, 2016.

[13] S. H. Dokhanchi, M. R. Bhavani Shankar, M. Alaee Kerahoodi., T. Stifter, and B. Ottersten, "Adaptive waveform design for automotive joint radar-communications system," in IEEE International Conference on Acoustics, Speech and Signal Processing (ICASSP), 2019.

[14] M. Bica, K. Huang, V. Koivunen, and U. Mitra, "Mutual information based radar waveform design for joint radar and cellular communication systems," in 2016 IEEE International Conference on Acoustics, Speech and Signal Processing (ICASSP), March 2016, pp. 3671-3675.

[15] C. A. Balanis, Antenna theory: analysis and design. John wiley \& sons, 2016.

[16] J. Qian, M. Lops, L. Zheng, X. Wang, and Z. He, "Joint system design for coexistence of MIMO radar and MIMO communication," IEEE Transactions on Signal Processing, vol. 66, no. 13, pp. 3504-3519, July 2018. 